\title{
SOBRE PROPOSICIONES A PRIORI Y CONTINGENTES Y A POSTERIORI Y NECESARIAS CONSIDERANDO TEOREMAS GEOMÉTRICOS ELEMENTALES Emilio Méndez Pinto*
}

RESUMEN: En este ensayo se exponen algunas consecuencias de las tesis esbozadas por Kripke ${ }^{1}$ sobre las verdades epistemológicas a priori y $a$ posteriori y su relación con las verdades metafísicas necesarias y contingentes considerando algunos teoremas geométricos elementales. Una exposición de este tipo es imposible, por principio, en el sistema kripkeano, por cuenta de contraejemplos que refutan tal imposibilidad.

PALABRAS ClAVE: Kripke, conocimiento a priori y a posteriori, geometrías euclidiana y no euclidiana.

RECEPCIÓN: 11 de septiembre de 2014.

ACEPTACIÓN: 9 de junio de 2015.

${ }^{1}$ Saul Kripke, El nombrar y la necesidad, 2005, México, Instituto de Investigaciones Filosóficas, UNAM, y también Kripke, Identidad y necesidad, 1978 México, Instituto de Investigaciones Filosóficas, UNAM.
* Politólogo del ITESM-CCM. 


\title{
SOBRE PROPOSICIONES A PRIORI Y CONTINGENTES Y A POSTERIORI Y NECESARIAS CONSIDERANDO TEOREMAS GEOMÉTRICOS ELEMENTALES
}

\author{
"La estimación de una teoría no está \\ simplemente determinada por su verdad." \\ GeORge Boole, \\ El análisis matemático de la lógica
}

Algunas nociones básicas. Recordemos brevemente, en aras de la exposición general, que los enunciados analíticos son aquellos en los que el predicado está contenido en el sujeto (no dicen nada sobre el mundo); los enunciados sintéticos son aquellos en los que el predicado no está contenido en el sujeto (dicen algo sobre el mundo); el conocimiento $a$ priori es el conocimiento (casi) ${ }^{1}$ completamente racional, independiente de la experiencia sensible, y el conocimiento a posteriori es el conocimiento no completamente racional, dependiente de la experiencia sensible.

Tradicionalmente, estas nociones estaban relacionadas en el siguiente

${ }^{1}$ Un conocimiento "completamente racional" es imposible por principio, porque todo conocimiento comienza con la experiencia sensible. sentido: si un juicio no dice nada sobre el mundo, entonces se debe a un conocimiento a priori; si un juicio dice algo sobre el mundo, entonces se debe a un conocimiento a posteriori. Se establecía, pues, una clara y aparentemente necesaria distinción entre los juicios analíticos y el conocimiento a priori, por un lado, y entre los juicios sintéticos y el conocimiento a posteriori, por el otro.

Es sabido que Kant fue el primero en romper esta clasificación y en establecer la posibilidad de juicios sintéticos a priori, esto es, juicios que dicen algo sobre el mundo y que son independientes de la experiencia sensible en la medida en que no se originan en ella (y no en la medida en 
que no comienzan con ella, dada la concepción kantiana de lo a priori). ${ }^{2}$

Más allá de las nociones básicas. Ahora bien, al hablar de esta revolución filosófica debe decirse que Kant conservó el prejuicio de que, si algo es necesario, entonces es un conocimiento a priori y de que, si algo es contingente, entonces es un conocimiento a posteriori. Este prejuicio es consistente con su filosofía porque para Kant el conocimiento a priori es conocimiento certero, imposible de ser contradicho por la experiencia. ${ }^{3}$ Kripke lo expone así:

Y, por supuesto, cuando Kant usa "necesario" para un tipo de proposición y "a priori" para un modo de conocimiento, no se le puede culpar de caer en la práctica común contemporánea de tratar a los dos términos como sinónimos intercambiables. Resulta claro, desde las primeras páginas de la Crítica, que la tesis de que el conocimiento de que algo es necesario tiene que ser un conocimiento a priori es considerada por Kant como una tesis importante y sustantiva, aunque obvia. ${ }^{4}$

Aquí mostraré que este prejuicio no es válido para por lo menos dos

${ }^{2}$ Immanuel Kant, Crítica de la razón pura, 2008, México, Porrúa, p. 27.

${ }^{3}$ RudolfCarnap, An introduction to the philosophy of science, 1995, Mineola, Dover, p. 177 y ss.

${ }^{4}$ Kripke, El nombrary la necesidad,pp. 156-7. casos, esto es, el caso que consideraremos como juicio a priori y contingente a la vez ${ }^{5}$ nos servirá para establecer la posibilidad, por lo menos para un caso, de juicios a posteriori y necesarios a la vez. También mostraré que una revisión del enunciado "Si un enunciado matemático es verdadero, entonces es necesario" produce que este sea falso para por lo menos un caso. ${ }^{6}$

El juicio que mostraremos como a priori y contingente a la vez será un teorema de la geometría euclidiana, paradigma de los juicios sintéti$\cos$ a priori y por tanto necesarios, según Kant. ${ }^{7}$ El principio de que la intuición no puede ser contradicha por la experiencia es, al igual que el principio que intercambia como sinónimos los términos "necesario" $\mathrm{y}$ "a priori", esencial para la filosofía kantiana. Pero el solo hecho de que

${ }^{5}$ Con el término "a la vez" me refiero a la noción de "al mismo tiempo"; y no solo para este caso, sino para todos los que siguen donde utilice tal término.

${ }^{6} \mathrm{La}$ afirmación de que si un enunciado matemático es verdadero, entonces es necesario la hace Kripke en la adenda de su conferencia El nombrar y la necesidad; véase p. 156. A lo largo de este ensayo, empero, también recurro a algunas tesis de Kripke con las que estoy plenamente de acuerdo, siendo la más evidente la que se refiere a la primera parte del título.

${ }^{7}$ Aquí únicamente consideraremos explícitamente una de las propiedades que, tradicionalmente, se le han dado al conocimiento a priori, a saber, la de ser necesario, y dejaremos fuera de consideración explícita la de ser, también, universal. 
los postulados de las geometrías no euclidianas sean formalmente verdaderos (es decir, no contradictorios) tira de golpe este principio, porque estas geometrías no euclidianas tienen un fuerte y sustantivo componente empírico. Es claro entonces que la intuición del espacio, elemento necesario para el entendimiento en la forma de una de las intuiciones sensibles a priori según Kant (la otra es el tiempo), pierde sus caracteres de necesidad y universalidad.

Antes de seguir es conveniente hacer tres aclaraciones generales.

En primer lugar, para nosotros la parte "sintética" del juicio bajo consideración será circunstancial, es decir, si al final resulta que, una vez demostrada la contingencia de tal juicio a priori, este es o no es sinté-

178 tico, cualquiera de las dos opciones es insignificante para la validez de nuestra tesis.

En segundo lugar, para que nuestra tesis sea lo más comprehensiva posible, es necesario que lo a priori lo entendamos no solo desde la definición clásica ofrecida al principio (es decir, un conocimiento a priori es aquel (casi) completamente racional, independiente de la experiencia sensible), sino también desde la siguiente definición: un juicio a priori es un juicio cuya verdad no requiere una demostración fáctica. Esta definición viene a decir que un juicio que pueda ser demostrado apriorísticamente no es un juicio que tenga que ser demostrado apriorísticamente. (Las razones explícitas de haber adoptado esta definición alterna de lo a priori serán evidentes más adelante.)

En tercer lugar, mostraremos que la noción de "contingente" que empleamos en nuestra primera tesis es válida para por lo menos dos posturas: para la aristotélica (lo contingente es falso en algún mundo posible) y para la leibniziana (lo contingente como aquello cuyo contrario es posible). Análogamente, la noción de "necesario" empleada en nuestra segunda tesis será igualmente válida para las mismas posturas: como aquello verdadero en todos los mundos posibles (Aristóteles) y como aquello cuyo contrario es imposible (Leibniz).

(Aquí emplearemos estas definiciones en un sentido disyuntivo inclusivo: lo contingente será aquello falso en algún mundo posible o aquello cuyo contrario es posible o ambas cosas, mientras que lo necesario será aquello verdadero en todos los mundos posibles o aquello cuyo contrario es imposible o ambas cosas.)

Sobre la primera tesis. La proposición que consideraremos para mostrar nuestra primera tesis, la posibilidad de proposiciones a priori y contingentes a la vez, es el teorema euclidiano que postulaba: "La suma de 
los ángulos internos de un triángulo es $=180^{\circ}$ ". El conocimiento sobre este juicio es a priori desde la definición clásica, es decir, es un conocimiento (casi) completamente racional, independiente de la experiencia sensible, y el enunciado "La suma de los ángulos internos de un triángulo es $=180^{\circ}$ " es a priori desde nuestra definición, es decir, es un juicio cuya verdad no requiere una demostración fáctica.

Ahora introduzcamos el postulado no euclidiano: "La suma de los ángulos internos de un triángulo no es $=180^{\circ}$. . Este postulado es verdadero para las geometrías elíptica (riemanniana) e hiperbólica (lobachevskiana) porque en el primer caso la suma de los ángulos internos de un triángulo es $>180^{\circ} \mathrm{y}$ en el segundo caso la suma de los ángulos internos de un triángulo es $<180^{\circ}$. Así pues, el contrario de "La suma de los ángulos internos de un triángulo es $=180^{\circ}$ ", esto es, "La suma de los ángulos internos de un triángulo no es $=180^{\circ}$ ", es posible, nuestro teorema euclidiano es falso en algún mundo posible, $y$ entonces es contingente.

Entonces la proposición "La suma de los ángulos internos de un triángulo es $=180^{\circ}$ " es a priori $y$ contingente a la vez. Además es claro que, considerando este caso, una revisión del enunciado "Si un enunciado matemático es verdadero, entonces es necesario" produce que este sea falso.

Sobre la segunda tesis. Para mostrar nuestra segunda tesis, la posibilidad de proposiciones a posteriori y necesarias a la vez, recurriremos al teorema siguiente: "En la geometría elíptica, la suma de los ángulos internos de un triángulo es $>180^{\circ}$ ". En este caso, el contrario de nuestro teorema, es decir, "En la geometría elíptica, la suma de los ángulos internos de un triángulo no es $>180^{\circ}$ ", es imposible, nuestro teorema es verdadero en todos los mundos posibles y entonces es necesario. Pero también es a posteriori, porque su verdad fue establecida a partir de una investigación (casi) ${ }^{8}$ puramente empírica sobre la estructura geométrica del espacio.

Entonces la proposición: "En la geometría elíptica, la suma de los ángulos internos de un triángulo es $>180^{\circ}$ " es a posteriori y necesaria a la vez. Además es claro que, considerando este caso, una revisión del enunciado "Si un enunciado matemático es verdadero, entonces es necesario" produce que este sea verdadero.

A partir de las segundas partes de nuestras conclusiones hasta el momento, es claro que la verdad "Si

${ }^{8}$ Una investigación "puramente empírica" es imposible por principio, porque en ella estaría ausente el carácter teleológico que tiene toda investigación. 
un enunciado matemático es verdadero, entonces es necesario" es irremediablemente contingente. Los únicos resquicios que podrían quedarles a quienes, por alguna u otra razón, se opongan a este desenlace tendrían que remitirse a los supuestos de que, o bien nuestro teorema geométrico euclidiano no es un enunciado matemático, o bien el contrario del teorema "La suma de los ángulos internos de un triángulo es $=180^{\circ}$ " es falso en todos los mundos posibles (es decir, es imposible).

Sobre nuestra definición de lo a priori. ${ }^{9}$ Antes dijimos, casi de pasada, que para nosotros un juicio a priori es aquel cuya verdad no requiere de una demostración fáctica. En realidad, las cuestiones y tesis que hemos expuesto hasta ahora pueden valerse, sin ningún problema, de la definición de lo a priori como un conocimiento (casi) completamente racional, independiente de la experiencia sensible. Sin embargo, hay casos en los que la aplicación de esta definición clásica resulta problemática, y es aquí en donde nuestra definición puede venir en auxilio.

En su ensayo Sobre las hipótesis que se encuentran en las bases de la geometria, Riemann ${ }^{10}$ sostiene que

${ }^{9}$ Para un desarrollo mucho más extenso y profundo, véase Kripke, El nombrar y la necesidad.

${ }^{10}$ Bernhard Riemann, "On the hypotheses which lie at the bases of geometry", en Stephen Hawking, God created the integers, 2007, Filadelfia, Running Press, p. 1031. el sistema euclidiano es un sistema de cuestiones de hechos: no es necesario, sino que posee una certeza empírica, hipotética. Esta perspectiva (o algunas parecidas) puede resultar problemática para la definición clásica de lo a priori. En efecto, no contempla la posibilidad de un conocimiento (ni siquiera casi) completamente racional e independiente de la experiencia sensible. La posición de Riemann supone, pues, la adopción de una perspectiva esencialmente empírica sobre la naturaleza de la geometría euclidiana, incompatible con la noción clásica de lo a priori.

Sin embargo, creo que tal posición (o algunas parecidas) no es incompatible con nuestra definición de lo a priori. Incluso dando por cierta la tesis de Riemann, y como consecuencia natural que el teorema euclidiano "La suma de los ángulos internos de un triángulo es $=180^{\circ}$ " surge de una investigación empírica sobre la estructura geométrica del espacio, sigue siendo cierto que la verdad del juicio "La suma de los ángulos internos de un triángulo es $=180^{\circ}$ " no requiere una demostración fáctica, es decir, su verdad es comprobable racionalmente.

Antes también dijimos que nuestra definición de lo a priori viene a decir, aunque sea incidentalmente, que una proposición que pueda ser demostrada apriorísticamente no es 
una proposición que tenga que ser demostrada apriorísticamente. En efecto, esto es lo que sucede con el juicio "La suma de los ángulos internos de un triángulo es $=180^{\circ}$ " si le damos un carácter a priori desde nuestra definición: uno puede demostrar a posteriori la verdad de este juicio si, por ejemplo, traza un triángulo equilátero en una hoja y suma sus ángulos internos, pero también sucede que su verdad puede comprobarse de manera esencialmente racional, es decir, apriorísticamente.

A modo de conclusión. En sus Fundamentos de la aritmética, Frege ${ }^{11}$ sostiene que las distinciones entre lo a priori y lo a posteriori y entre lo analítico y lo sintético no tienen que ver con el contenido del juicio, sino con la justificación para hacer el juicio.

Si nosotros definimos un juicio a priori como aquel cuya verdad no requiere una demostración fáctica, entonces es claro que un juicio a posteriori será aquel cuya verdad requiere una demostración fáctica.

Nuestra justificación para decir que un juicio es a priori no involucra el posible hecho de que el conocimiento sobre tal juicio sea independiente de la experiencia sensible en uno $u$ otro grado. En efecto, no nos intere-

${ }^{11}$ Gottlob Frege, The foundations of arithmetic. A logico-mathematical enquiry into the concept of number, 1992, Northwestern University Press, Evanston, pp. 3-4. san las condiciones psicológicas (o fisiológicas, físicas) de un juicio particular, sino la ausencia o presencia de un elemento fáctico en él: en un conocimiento a posteriori hay un elemento fáctico que impide que la verdad de tal juicio sea demostrada (comprobada) a partir de procedimientos puramente racionales, mientras que en un conocimiento a priori no hay un elemento fáctico que impida que su verdad sea demostrada (comprobada) a partir de procedimientos puramente racionales.

Es así que nuestra posición es similar a la de Frege: para que una verdad sea a posteriori, debe ser imposible construir una prueba de ella sin incluir una apelación a los hechos, mientras que para que una verdad sea a priori debe ser posible derivar su prueba exclusivamente de leyes generales que por sí mismas no necesitan o no admiten prueba alguna.

Quizá esta conclusión sea mucho más clara si consideramos las siguientes dos ilustraciones, una para el caso de lo a priori y otra para el caso de lo a posteriori.

1) Arriba dijimos, junto con Frege, que para que una verdad sea a priori debe ser posible derivar su prueba exclusivamente de leyes generales que por sí mismas no necesitan o no admiten prueba alguna. Nuestro juicio "La suma de los ángulos internos de un 
triángulo es $=180^{\circ}$ " cumple con los requisitos, por así decirlo, para ser una verdad a priori en este sentido, es decir, sucede que sus leyes generales no necesitan pero sí admiten prueba alguna (uno puede comprobar fácticamente este juicio, como ya vimos con el ejemplo del triángulo equilátero trazado sobre una hoja).

2) Si un juicio a posteriori es aquel cuya verdad requiere una demostración fáctica, o si para que una verdad sea a posteriori debe ser imposible construir una prueba de ella sin incluir una apelación a los hechos, entonces es claro que desde nuestra posición todas las proposiciones de las ciencias naturales son a posteriori. En la filosofía kantiana todas las proposiciones científicas son juicios sintéticos a priori, pero esto es porque Kant se ocupó del contenido de los juicios, y no de la justificación para hacerlos. 